\title{
Avaliação da Resistência de Isolinhas de Feijoeiro a Diferentes Patótipos de Colletotrichum lindemuthianum, Uromyces appendiculatus e Phaeoisariopsis griseola*
}

\author{
Vilmar Antonio Ragagnin ${ }^{1,2}$, Ana Lília Alzate-Marin ${ }^{1,3}$, Thiago Livio P. O. Souza ${ }^{1}$, Klever Márcio A. \\ Arruda $^{1}$, Maurilio A. Moreira ${ }^{1,4}$ \& Everaldo G. Barros ${ }^{1,5}$ \\ ${ }^{1}$ Instituto de Biotecnologia Aplicada à Agropecuária - BIOAGRO/Universidade Federal de Viçosa (UFV); ${ }^{2}$ e-mail: \\ vilmar@ alunos.ufv.br; ${ }^{3}$ e-mail:aalzate @ alunos.ufv.br; ${ }^{4}$ Departamento de Bioquímica e Biologia Molecular/UFV, e-mail: \\ moreira@ufv.br, ${ }^{5}$ Departamento de Biologia Geral/UFV, e-mail: ebarros@ufv.br, CEP 36571-000, Viçosa, MG, fax (31) 3899-2864.
}

(Aceito para publicação em 24/09/2003)

Autor para correspondência: Everaldo G. de Barros

RAGAGNIN, V.A., ALZATE-MARIN, A.L., SOUZA, T.L.P.O., ARRUDA, K.M.A., MOREIRA, M.A. \& BARROS, E.G. Avaliação da resistência de isolinhas de feijoeiro ao Colletotrichum lindemuthianum, Uromyces appendiculatus e Phaeoisariopsis griseola. Fitopatologia Brasileira 28:591-596. 2003.

\section{RESUMO}

Isolinhas de feijoeiro (Phaseolus vulgaris) derivadas da cultivar Rudá, com grãos do tipo "carioca", contendo os genes de resistência à antracnose (Co-4 do cv. TO ou Co-6 do cv. AB 136 ou Co-10 do cv. Ouro Negro), ferrugem (gene do cv. Ouro Negro) e mancha-angular (phg-1 da linhagem AND 277) foram desenvolvidas pelo Programa de Melhoramento do Feijoeiro do BIOAGRO/UFV, para serem utilizadas na piramidação desses genes em uma única cultivar. Este trabalho teve como objetivo determinar o espectro de resistência dessas isolinhas a diferentes patótipos de Colletotrichum lindemuthianum, Uromyces appendiculatus e Phaeoisariopsis griseola, visando selecionar as linhagens mais similares aos progenitores doadores quanto à sua resistência. Para isto, foram conduzidas inoculações artificiais com 18 patótipos de $C$. lindemuthianum, dez patótipos de $U$. appendiculatus e quatro patótipos de $P$. griseola. Para resistência à antracnose, foi selecionada uma linhagem do cruzamento Rudá x TO, homozigota resistente a 17 patótipos, uma linhagem do retrocruzamento Rudá x AB 136, homozigota resistente a 15 patótipos e uma linhagem do cruzamento Rudá x Ouro Negro, homozigota resistente a 15 patótipos de $C$. lindemuthianum. Para resistência à ferrugem, uma linhagem do cruzamento Rudá x Ouro Negro, apresentou-se homozigota resistente aos dez patótipos de $U$. appendiculatus testados. Para resistência à mancha-angular, uma linhagem do cruzamento Rudá x AND277 apresentou-se homozigota resistente aos quatro patótipos de $P$. griseola inoculados. As melhores isolinhas estão sendo intercruzadas visando a piramidação de genes de resistência à ferrugem, à antracnose e à mancha-angular e o desenvolvimento de cultivares essencialmente derivadas da cultivar Rudá.

Palavras-chave adicionais: Phaseolus vulgaris, resistência múltipla, piramidação.

\section{ABSTRACT}

Evaluation of resistance of common bean near isogenic lines to Colletotrichum lindemuthianum, Uromyces appendiculatus and Phaeoisariopsis griseola

Common bean (Phaseolus vulgaris) near isogenic lines (NIL's) derived from the "carioca-type" cultivar Rudá harboring resistance genes to anthracnose ( $\mathrm{Co}-4$ from cv. TO or $\mathrm{Co}-6$ from cv. AB 136 or $C o-10$ from cv. Ouro Negro), rust (gene from cv. Ouro Negro) and angular leaf spot (phg-1 from the line AND 277) were developed by the Bean Breeding Program of BIOAGRO/UFV (Minas Gerais, Brazil) to be used for gene pyramiding. This work had the objective of determining the resistance spectra of these NIL's to different pathotypes of Colletotrichum lindemuthianum, Uromyces appendiculatus and Phaeoisariopsis griseola in order to select the lines most similar to those of the donor parents with regards to resistance. Artificial inoculations were conducted using 18, ten and four pathotypes of $C$. lindemuthianum, $U$. appendiculatus, and P. griseola, respectively. For anthracnose resistance, the following lines were selected: one line resistant to 17 pathotypes derived from the cross Rudá $\mathrm{x}$ TO, one line resistant to 15 pathotypes derived from the cross Rudá x AB 136, and one line resistant to 15 pathotypes derived from the cross Rudá x Ouro Negro. One line derived from the cross Rudá x Ouro Negro was resistant to ten $U$. appendiculatus pathotypes. One line derived from the cross Rudá x AND277 was resistant to the four $P$. griseola pathotypes tested. These NIL's are now being intercrossed to pyramid the resistance genes to rust, anthracnose and angular leaf spot in the "carioca-type" background Rudá.

\section{INTRODUÇÃO}

Vários problemas sanitários afetam a cultura do feijoeiro comum (Phaseolus vulgaris L.). Doenças foliares *Parte da tese de mestrado do primeiro autor. Universidade Federal de
Viçosa (2001). como a ferrugem, antracnose e mancha-angular, causadas respectivamente por Uromyces appendiculatus (Pers.) Unger var. appendiculatus, Colletotrichum lindemuthianum (Sacc. \& Magn.) Scribner e Phaeoisariopsis griseola (Sacc.) Ferraris, encontram-se entre as principais responsáveis por prejuízos na produção de grãos do feijoeiro. Lanza et al. (1997) e Faleiro 
et al. (2001b) estudaram o comportamento de cultivares comerciais de feijoeiro quanto à resistência/suscetibilidade a esses três patógenos e concluíram que a maioria das cultivares comerciais testadas foi suscetível.

No programa de melhoramento do BIOAGRO/,foi observado que a cultivar Ouro Negro (acesso Honduras 35) apresentou resistência a 14 patótipos de $U$. appendiculatus e a vários patótipos de C. lindemuthianum (Faleiro, 1997). Utilizando a técnica de inoculação múltipla com os patótipos 2, 8, 10 e 11 de $U$. appendiculatus, evidenciou-se que a resistência à ferrugem, presente em 'Ouro Negro', é conferida por um bloco gênico (Corrêa, 1999). Por meio da técnica de inoculações seqüenciais, com os patótipos 73 e 89 de $C$. lindemuthianum e os patótipos 2, 8, 10 e 11 de $U$. appendiculatus, demonstrou-se que os genes de resistência a estes patógenos estão ligados a uma distância de $12,3 \mathrm{cM}$ (Corrêa, 1999; Faleiro et al., 2000).

Entre as principais fontes de resistência à antracnose do feijoeiro encontram-se as cultivares TO (fonte do gene Co-4) e AB 136 (fonte do gene Co-6) (Schwartz et al., 1982, Bassett, 1996). Estas cultivares fazem parte das 12 diferenciadoras internacionais para antracnose. No Brasil, a cultivar AB 136 apresentou resistência a 35 patótipos identificados até o presente e a cultivar TO apresentou resistência a 31 deles, sendo suscetível aos patótipos 320, 339, 343 e 453 (Rava et al., 1994; Andrade et al., 1999; Thomazella et al., 2000).

Com relação à mancha-angular, as linhagens AND277, MAR-2, México 54, BAT 332 e Cornell 49-242 são importantes fontes de resistência, sendo de grande interesse pelo Programa de Melhoramento do Feijoeiro do BIOAGRO/ UFV. No estudo realizado por Nietsche (1997), a cultivar AND 277 , de origem andina, mostrou-se resistente a nove dos 13 patótipos de $P$. griseola testados, apresentando resistência aos patótipos mais freqüentemente encontrados no Estado de Minas Gerais. Carvalho et al. (1998) identificaram um gene de resistência à mancha-angular em AND-277, o qual designaram Phg-1.

A introgressão de genes de resistência em uma cultivar-elite (piramidação) é uma estratégia que vem sendo adotada em programas de melhoramento de plantas autógamas (Hittalmani et al., 2000). O método a ser escolhido para piramidação depende do tipo de resistência com a qual se está trabalhando. No método de transferência paralela, os genes de resistência individuais são incorporados por retrocruzamentos em diversas isolinhas, as quais são, em seguida, intercruzadas.

Uma das etapas importantes no processo de piramidação dos genes de resistência é a caracterização fenotípica das isolinhas obtidas nos vários subprogramas. Esta é feita por inoculação de patótipos dos diferentes patógenos de interesse. As informações assim obtidas servem como base para a seleção das isolinhas com maior espectro de resistência que serão usadas na etapa de intercruzamentos.

Nos últimos anos, o Programa de Melhoramento do Feijoeiro do BIOAGRO/UFV tem-se concentrado na obtenção de isolinhas resistentes à antracnose, ferrugem e manchaangular. Assim, como continuação deste programa, o presente trabalho teve como objetivo a caracterização de 18 isolinhas quanto à resistência/suscetibilidade a patótipos de $C$. lindemuthianum, U. appendiculatus e $P$. griseola, respectivamente, visando selecionar aquelas com maior espectro de resistência para posteriores intercruzamentos.

\section{MATERIALEMÉTODOS}

Neste trabalho foram avaliadas 18 isolinhas de feijoeiro com grãos do tipo "carioca" obtidas em quatro subprogramas de retrocruzamentos envolvendo a cultivar suscetível Rudá (usado como progenitor recorrente) e fontes de resistência à antracnose (TO, AB 136 e Ouro Negro), ferrugem (Ouro Negro) e mancha-angular (AND 277). Os retrocruzamentos foram monitorados por marcadores moleculares de forma a garantir a recuperação do genoma do progenitor recorrente. Foram feitos três retrocruzamentos no subprograma envolvendo a cultivar Ouro Negro e quatro retrocruzamentos nos restantes. Todas as isolinhas estavam nas gerações RC3F3 ou RC4F3. A cultivar Rudá foi desenvolvida pelo CIAT, a partir do cruzamento entre as cultivares Carioca e Rio Tibagi, tendo sido introduzido no Brasil como linhagem A 285, pela EMBRAPA Arroz e Feijão (Goiânia, GO). As cultivares TO e AB 136 contêm os genes $C o-4$ e $C o-6$ de resistência à antracnose, respectivamente. A cultivar Ouro Negro possui um gene ou bloco gênico de resistência à ferrugem e um gene de resistência à antracnose tentativamente designado Co10 (Alzate-Marin et al., 2001). A cultivar AND 277 contêm o gene phg-l de resistência à mancha-angular. As isolinhas, objeto deste estudo, foram obtidas pelo Programa de Melhoramento do Feijoeiro do BIOAGRO/UFV (Alzate-Marin et al., 1997; Faleiro et al., 2001a).

Para avaliar a resistência/suscetibilidade das isolinhas de feijoeiro à ferrugem foram cultivadas dez sementes de cada uma das 18 isolinhas e de seus respectivos progenitores para cada um dos patótipos de $U$. appendiculatus testados. As sementes foram semeadas diretamente em bandejas plásticas contendo uma mistura de solo e esterco curtido, na proporção de $4: 1$, adubada no momento do preparo com $5 \mathrm{~kg}$ do adubo 4-14-8 por $\mathrm{m}^{3}$ de substrato. As plantas foram mantidas em casa de vegetação até o momento da inoculação.

Os patótipos 32, 45, 46, 47, 49, 50, 52, 54, 58 e 59 de $U$. appendiculatus utilizados foram os identificados e classificados por Faleiro et al. (1999a). As culturas monospóricas, armazenadas a $5^{\circ} \mathrm{C}$ e $50 \%$ de umidade relativa, foram multiplicadas no hospedeiro suscetível US Pinto 111 antes da inoculação, visando recuperar a viabilidade dos uredósporos. A inoculação foi realizada quando as folhas primárias apresentavam aproximadamente $2 / 3$ do seu desenvolvimento completo, cerca de dez dias após a semeadura. Os uredósporos, na concentração de 2,0 x $10^{4}$ esporos/ml (Davison \& Vaughan, 1964), foram suspensos em água destilada contendo $0,05 \%$ de Tween 20 e aspergidos em ambas as superfícies foliares, com o auxílio de um atomizador 
De Vilbiss nำ 15, acionado por um compressor elétrico (Carrijo et al., 1980). Após a inoculação e rápida secagem ao ar, as plantas foram transferidas para câmara de nevoeiro $\left(20 \pm 1^{\circ} \mathrm{C}\right.$ e umidade relativa $>95 \%$ ), onde permaneceram por $48 \mathrm{~h}$, sob fotoperíodo de $12 \mathrm{~h}$. Após esse período, foram novamente transferidas para a casa de vegetação $\left(20 \pm 5^{\circ} \mathrm{C}\right)$, onde permaneceram até serem avaliadas.

$\mathrm{Na}$ avaliação dos sintomas de ferrugem foram considerados seis graus de reação segundo a escala proposta no "The Bean Rust Workshop" (Stavely et al., 1983). As plantas que apresentaram graus de 1 a 3 foram consideradas resistentes e as com grau 4 ou maior, suscetíveis. O grau foi determinado mediante a observação visual das pústulas na face superior das folhas primárias.

A caracterização da resistência à antracnose foi realizada utilizando dez sementes de cada progenitor e de cada uma das 15 isolinhas obtidas dos cruzamentos Rudá $\mathrm{x}$ TO (quatro isolinhas), Rudá x AB 136 (seis isolinhas) e Rudá x Ouro Negro (cinco isolinhas) para cada patótipo de $C$. lindemuthianum testado. Os patótipos 7, 23, 55, 64, 65, 67, 73, 79, 81, 83, 87, 89, 95, 102, 117, 119, 343 e 453 de $C$. lindemuthianum testados foram aqueles caracterizados por Rava et al. (1994). As culturas monospóricas originais dos patótipos utilizados foram cedidas pela EMBRAPA Arroz e Feijão. O preparo do inóculo e a inoculação seguiram a metodologia adaptada de Pio-Ribeiro \& Chaves (1975). Para a obtenção de inóculo, cada patótipo foi cultivado durante dez dias a $23{ }^{\circ} \mathrm{C}$ em tubos de ensaio contendo vagens esterilizadas e parcialmente imersas em meio ágar-água.

O preparo do solo, a adubação e a condução das plantas até o momento da inoculação foram semelhantes aos do ensaio com ferrugem. A inoculação foi feita dez dias após o plantio, utilizando-se uma suspensão contendo $1,2 \times 10^{6}$ conídios/ml, a qual foi aplicada em ambas as superfícies das folhas primárias, com o auxílio de um atomizador De Vilbiss no 15 acionado por um compressor elétrico. Após a inoculação, as plantas foram incubadas por cinco dias na câmara de nevoeiro $\left(20 \pm 1{ }^{\circ} \mathrm{C}\right.$ e $>95 \%$ de umidade relativa), sob fotoperíodo de $12 \mathrm{~h}$. Após este período, foi realizada a avaliação dos sintomas de antracnose com base na escala de 1 a 9 descrita por Rava et al. (1993). As plantas que apresentaram graus de reação de 1 a 3 foram consideradas resistentes e aquelas com grau 4 ou maior, suscetíveis.

A caracterização da resistência à mancha-angular foi realizada utilizando dez sementes de cada progenitor e de cada uma das isolinhas provenientes dos cruzamentos Rudá x AND 277 (três isolinhas) e Rudá x Ouro Negro (cinco isolinhas) para cada patótipo de $P$. griseola testado. As sementes foram semeadas em vasos plásticos de 2,5 L, três por vaso. O preparo do solo, a adubação e a condução das plantas até o momento da inoculação foram semelhantes aos do ensaio com ferrugem.

Para avaliação da resistência à mancha-angular, foram utilizados os patótipos 31-15, 63-19, 63-23 e 63-31, classificados por Nietsche (1997). O inóculo de cada patótipo foi reproduzido em placas de Petri contendo meio V8. A inoculação foi realizada após o aparecimento da primeira folha trifoliolada em ambas as superfícies da folha com uma suspensão do patógeno, previamente preparada e ajustada para concentração de $2 \times 10^{4}$ conídios $/ \mathrm{ml}$. Os procedimentos de inoculação e transferência para a câmara de nevoeiro e para a casa de vegetação foram idênticos aos realizados no ensaio de ferrugem. A severidade da doença foi avaliada visualmente aos 18 e 25 dias após a inoculação, utilizando uma escala com nove graus de severidade baseada em Van Schoonhoven \& Pastor-Corrales (1987), descrita a seguir: 1plantas sem sintomas da doença; 2 - presença de até $3 \%$ de lesões; 3- presença de até 5\% de lesões foliares, sem esporulação do patógeno; 4- presença de lesões esporuladas cobrindo $10 \%$ da área foliar; 5- presença de várias lesões esporuladas entre 2 e $3 \mathrm{~mm}$, cobrindo $10-15 \%$ da área foliar; 6- presença de numerosas lesões esporuladas maiores que 3 $\mathrm{mm}$, cobrindo entre $15-20 \%$ da área foliar; 7- presença de numerosas lesões esporuladas maiores que $3 \mathrm{~mm}$, cobrindo entre 20-25\% da área foliar; 8- presença de numerosas lesões esporuladas maiores que $3 \mathrm{~mm}$, que cobrem entre $25-30 \%$ da área foliar, associadas a tecidos; e 9- sintomas severos da doença, resultando em queda prematura de folhas e morte da planta. As plantas que apresentaram graus 1 a 3 foram consideradas resistentes e as com grau 4 ou maior, suscetíveis.

\section{RESULTADOS E DISCUSSÃO}

A inoculação das isolinhas e dos progenitores usados como fontes de resistência foi conduzida para avaliar a resistência/suscetibilidade à antracnose, ferrugem e manchaangular. Semelhantemente aos resultados obtidos por Rava et al. (1994), as cultivares TO e AB 136 mostraram-se resistentes a 17 e 18 patótipos de $C$. lindemuthianum, respectivamente. A cultivar TO foi suscetível apenas ao patótipo 343. A cultivar Ouro Negro apresentou-se homozigota resistente a dez dos 18 patótipos testados, indicando que é uma cultivar de amplo espectro de resistência à antracnose (Tabela 1). Mostrou-se, no entanto, suscetível ao patótipo 65 e comportou-se como uma multilinha frente a sete dos 18 patótipos testados. Neste caso, algumas plantas mostraram resistência e outras suscetibilidade a um mesmo patótipo testado. Tal fato foi também observado em 'Ouro Negro’ por Faria et al. (2000) quando avaliaram essa cultivar para resistência ao vírus do mosaico comum necrótico (Bean common mosaic virus, BCMV). Trabalhos de alelismo recentes mostraram que o gene de resistência à antracnose da cultivar Ouro Negro é um novo gene identificado tentativamente designado como Co-10 (Alzate-Marin et al., 2001). Com relação à ferrugem, as cultivares $\mathrm{TO}, \mathrm{AB} 136 \mathrm{e}$ AND 277 foram suscetíveis a todos os patótipos de $U$. appendiculatus testados, enquanto a cultivar Ouro Negro apresentou resistência a todos eles. Faleiro et al. (1999b) já haviam destacado a importância da cultivar Ouro Negro como fonte doadora de genes de resistência à ferrugem em trabalhos de melhoramento. Com relação à mancha-angular, a cultivar Ouro 
V.A. Ragagnin et al.

TABELA 1 - Avaliação da resistência à ferrugem em cultivares e isolinhas de feijoeiro comum (Phaseolus vulgaris)

\begin{tabular}{|c|c|c|c|c|c|c|c|c|c|c|}
\hline \multirow{2}{*}{$\begin{array}{l}\text { Genótipo de } \\
\text { Phaseolus vulgaris }\end{array}$} & \multicolumn{10}{|c|}{ Patótipos de Uromyces appendiculatus* } \\
\hline & 54 & 49 & 58 & 52 & 59 & 45 & 46 & 50 & 47 & 32 \\
\hline Rudá & $\mathrm{rr}$ & $\mathrm{rr}$ & $\mathrm{rr}$ & $\mathrm{rr}$ & $\mathrm{rr}$ & $\mathrm{rr}$ & $\mathrm{rr}$ & $\mathrm{rr}$ & $\mathrm{rr}$ & $\mathrm{rr}$ \\
\hline Ouro Negro & RR & RR & RR & RR & RR & $\mathrm{RR}$ & RR & RR & RR & RR \\
\hline $\mathrm{TO}$ & $\mathrm{rr}$ & $\mathrm{rr}$ & $\mathrm{rr}$ & $\mathrm{rr}$ & $\mathrm{rr}$ & rr & $\mathrm{rr}$ & $\mathrm{rr}$ & $\mathrm{rr}$ & $\mathrm{rr}$ \\
\hline AB 136 & $\mathrm{rr}$ & $\mathrm{rr}$ & $\mathrm{rr}$ & $\mathrm{rr}$ & $\mathrm{rr}$ & $\mathrm{rr}$ & $\mathrm{rr}$ & $\mathrm{rr}$ & $\mathrm{rr}$ & $\mathrm{rr}$ \\
\hline AND 277 & $\mathrm{rr}$ & $\mathrm{rr}$ & $\mathrm{rr}$ & $\mathrm{rr}$ & $\mathrm{rr}$ & $\mathrm{rr}$ & $\mathrm{rr}$ & $\mathrm{rr}$ & $\mathrm{rr}$ & $\mathrm{rr}$ \\
\hline ON-06-99 & RR & $\mathrm{RR}$ & $\mathrm{RR}$ & RR & $\mathrm{RR}$ & $\mathrm{RR}$ & $\mathrm{RR}$ & $\mathrm{RR}$ & RR & $\mathrm{R} / \mathrm{r}$ \\
\hline ON-25-99 & RR & RR & RR & RR & RR & RR & RR & RR & RR & RR \\
\hline ON-43-99 & $\mathrm{R} / \mathrm{r}$ & $\mathrm{R} / \mathrm{r}$ & $\mathrm{R} / \mathrm{r}$ & $\mathrm{R} / \mathrm{r}$ & $\mathrm{R} / \mathrm{r}$ & $\mathrm{R} / \mathrm{r}$ & $\mathrm{R} / \mathrm{r}$ & $\mathrm{R} / \mathrm{r}$ & $\mathrm{R} / \mathrm{r}$ & $\mathrm{R} / \mathrm{r}$ \\
\hline ON-45-99 & $\mathrm{R} / \mathrm{r}$ & $\mathrm{R} / \mathrm{r}$ & $\mathrm{R} / \mathrm{r}$ & $\mathrm{R} / \mathrm{r}$ & $\mathrm{R} / \mathrm{r}$ & $\mathrm{R} / \mathrm{r}$ & $\mathrm{R} / \mathrm{r}$ & RR & $\mathrm{R} / \mathrm{r}$ & $\mathrm{R} / \mathrm{r}$ \\
\hline ON-48-99 & RR & RR & $\mathrm{RR}$ & RR & RR & $\mathrm{RR}$ & $\mathrm{RR}$ & $\mathrm{RR}$ & RR & $\mathrm{RR}$ \\
\hline TO-41-5-1-1 & $\mathrm{rr}$ & $\mathrm{rr}$ & $\mathrm{rr}$ & $\mathrm{rr}$ & $\mathrm{rr}$ & $\mathrm{rr}$ & $\mathrm{rr}$ & $\mathrm{rr}$ & $\mathrm{rr}$ & $\mathrm{rr}$ \\
\hline TO-41-5-2-5 & $\mathrm{rr}$ & $\mathrm{rr}$ & $\mathrm{rr}$ & $\mathrm{rr}$ & $\mathrm{rr}$ & $\mathrm{rr}$ & $\mathrm{rr}$ & $\mathrm{rr}$ & $\mathrm{rr}$ & $\mathrm{rr}$ \\
\hline TO-41-5-6-4 & $\mathrm{rr}$ & $\mathrm{rr}$ & $\mathrm{rr}$ & $\mathrm{rr}$ & $\mathrm{rr}$ & $\mathrm{rr}$ & $\mathrm{rr}$ & $\mathrm{rr}$ & $\mathrm{rr}$ & $\mathrm{rr}$ \\
\hline TO-41-5-6-24 & $\mathrm{rr}$ & $\mathrm{rr}$ & $\mathrm{rr}$ & $\mathrm{rr}$ & $\mathrm{rr}$ & $\mathrm{rr}$ & $\mathrm{rr}$ & $\mathrm{rr}$ & $\mathrm{rr}$ & $\mathrm{rr}$ \\
\hline AB-74-1-3 & $\mathrm{rr}$ & $\mathrm{rr}$ & $\mathrm{rr}$ & $\mathrm{rr}$ & $\mathrm{rr}$ & $\mathrm{rr}$ & $\mathrm{rr}$ & $\mathrm{rr}$ & $\mathrm{rr}$ & $\mathrm{rr}$ \\
\hline AB-74-1-9 & $\mathrm{rr}$ & $\mathrm{rr}$ & $\mathrm{rr}$ & $\mathrm{rr}$ & $\mathrm{rr}$ & $\mathrm{rr}$ & $\mathrm{rr}$ & $\mathrm{rr}$ & $\mathrm{rr}$ & $\mathrm{rr}$ \\
\hline AB-74-1-10 & $\mathrm{rr}$ & $\mathrm{rr}$ & $\mathrm{rr}$ & $\mathrm{rr}$ & $\mathrm{rr}$ & $\mathrm{rr}$ & $\mathrm{rr}$ & $\mathrm{rr}$ & $\mathrm{rr}$ & $\mathrm{rr}$ \\
\hline AB-74-1-13 & $\mathrm{rr}$ & $\mathrm{rr}$ & $\mathrm{rr}$ & $\mathrm{rr}$ & $\mathrm{rr}$ & $\mathrm{rr}$ & $\mathrm{rr}$ & $\mathrm{rr}$ & $\mathrm{rr}$ & $\mathrm{rr}$ \\
\hline AB-74-1-16 & $\mathrm{rr}$ & $\mathrm{rr}$ & $\mathrm{rr}$ & $\mathrm{rr}$ & $\mathrm{rr}$ & $\mathrm{rr}$ & $\mathrm{rr}$ & $\mathrm{rr}$ & $\mathrm{rr}$ & $\mathrm{rr}$ \\
\hline AB-74-1-18 & $\mathrm{rr}$ & $\mathrm{rr}$ & $\mathrm{rr}$ & $\mathrm{rr}$ & $\mathrm{rr}$ & $\mathrm{rr}$ & $\mathrm{rr}$ & $\mathrm{rr}$ & $\mathrm{rr}$ & $\mathrm{rr}$ \\
\hline AND-7 2-9-4-6 & $\mathrm{rr}$ & $\mathrm{rr}$ & $\mathrm{rr}$ & $\mathrm{rr}$ & $\mathrm{rr}$ & rr & $\mathrm{rr}$ & $\mathrm{rr}$ & $\mathrm{rr}$ & $\mathrm{r}$ \\
\hline AND-7-2-9-4-10 & $\mathrm{rr}$ & $\mathrm{rr}$ & $\mathrm{rr}$ & $\mathrm{rr}$ & $\mathrm{rr}$ & $\mathrm{rr}$ & $\mathrm{rr}$ & $\mathrm{rr}$ & $\mathrm{rr}$ & $\mathrm{rr}$ \\
\hline AND-7-2-9-7-10 & $\mathrm{rr}$ & $\mathrm{rr}$ & $\mathrm{rr}$ & $\mathrm{rr}$ & $\mathrm{rr}$ & $\mathrm{rr}$ & $\mathrm{rr}$ & $\mathrm{rr}$ & $\mathrm{rr}$ & $\mathrm{rr}$ \\
\hline
\end{tabular}

*Designação dos patótipos atribuída por Faleiro et al. (1999a); RR homozigoto resistente; rr - homozigoto suscetível; R/r - em segregação.
Negro apresentou-se resistente a dois patótipos testados e segregou quanto à resistência a outros dois patótipos. A linhagem AND 277 comportou-se como resistente aos quatro patótipos de $P$. griseola testados. Trabalhos anteriores já demonstraram que esta cultivar é uma excelente fonte de resistência à mancha-angular (Nietsche, 1997; Carvalho et al., 1998).

A cultivar Rudá, utilizada como progenitor recorrente, foi também caracterizada quanto à resistência/suscetibilidade aos patótipos de antracnose, ferrugem e mancha-angular. Em relação à antracnose, Rudá comportou-se como resistente aos patótipos 23, 64 e 119, segregou quanto à resistência aos patótipos 102 e 343 e foi suscetível a 13 dos 18 patótipos de C. lindemuthianum testados. Quanto à ferrugem e à manchaangular, a cultivar Rudá foi suscetível a todos os patótipos testados.

Dos resultados da reação de resistência das isolinhas à antracnose, verificou-se que a isolinha TO-41-5-6-24 comportouse como homozigota resistente a 17 patótipos e como segregante frente ao patótipo 343. A isolinha AB-74-1-18 comportou-se como homozigota resistente a 15 dos 18 patótipos testados e como segregante frente aos patótipos 64, 119 e 343 . A isolinha ON25-99 apresentou-se homozigota resistente a 15 patótipos de $C$. lindemuthianum, segregante frente ao patótipo 87 e suscetível aos patótipos 64 e 65 (Tabela 2).

Quanto à avaliação da resistência à ferrugem, verificou-se que as isolinhas ON-25-99 e ON-48-99 resultantes do retrocruzamento entre as cultivares Rudá e Ouro Negro comportaram-se como homozigotas resistentes a todos os

TABELA 2 - Avaliação da resistência à antracnose em cultivares e isolinhas de feijoeiro-comum (Phaseolus vulgaris)

\begin{tabular}{|c|c|c|c|c|c|c|c|c|c|c|c|c|c|c|c|c|c|c|}
\hline \multirow{2}{*}{$\begin{array}{l}\text { Genótipo de } \\
\text { Phaseolus vulgaris }\end{array}$} & \multicolumn{18}{|c|}{ Patótipos de Colletotrichum lindemuthianum } \\
\hline & 7 & 23 & 55 & 64 & 65 & 67 & 73 & 79 & 81 & 83 & 87 & 89 & 95 & 102 & 117 & 119 & 343 & 453 \\
\hline $\begin{array}{l}\text { Rudá } \\
\end{array}$ & $\mathrm{rr}$ & RR & rr & RR & $\mathrm{rr}$ & rr & $\mathrm{rr}$ & $\mathrm{rr}$ & $\mathrm{rr}$ & $\mathrm{rr}$ & $\mathrm{rr}$ & $\mathrm{rr}$ & $\mathrm{rr}$ & $\mathrm{R} / \mathrm{r}$ & $\mathrm{rr}$ & RR & $\mathrm{R} / \mathrm{rr}$ & $\mathrm{rr}$ \\
\hline Ouro Negro & $\mathrm{R} / \mathrm{r}$ & $\mathrm{RR}$ & RR & $\mathrm{R} / \mathrm{r}$ & $\mathrm{rr}$ & $\mathrm{RR}$ & $\mathrm{RR}$ & $\mathrm{RR}$ & RR & $\mathrm{R} / \mathrm{r}$ & $\mathrm{RR}$ & RR & $\mathrm{R} / \mathrm{r}$ & $\mathrm{RR}$ & $\mathrm{R} / \mathrm{r}$ & $\mathrm{RR}$ & $\mathrm{R} / \mathrm{r}$ & $\mathrm{R} / \mathrm{r}$ \\
\hline TO & $\mathrm{RR}$ & $\mathrm{RR}$ & $\mathrm{RR}$ & $\mathrm{RR}$ & $\mathrm{RR}$ & $\mathrm{RR}$ & RR & $\mathrm{RR}$ & $\mathrm{RR}$ & $\mathrm{RR}$ & $\mathrm{RR}$ & $\mathrm{RR}$ & $\mathrm{RR}$ & $\mathrm{RR}$ & $\mathrm{RR}$ & $\mathrm{RR}$ & $\mathrm{rr}$ & $\mathrm{RR}$ \\
\hline $\mathrm{AB} 136$ & $\mathrm{RR}$ & $\mathrm{RR}$ & $\mathrm{RR}$ & $\mathrm{RR}$ & $\mathrm{RR}$ & $\mathrm{RR}$ & $\mathrm{RR}$ & RR & $\mathrm{RR}$ & $\mathrm{RR}$ & $\mathrm{RR}$ & RR & $\mathrm{RR}$ & $\mathrm{RR}$ & $\mathrm{RR}$ & $\mathrm{RR}$ & $\mathrm{RR}$ & RR \\
\hline ON-06-99 & RR & $\mathrm{RR}$ & $\mathrm{RR}$ & $\mathrm{R} / \mathrm{r}$ & $\mathrm{rr}$ & $\mathrm{RR}$ & RR & RR & $\mathrm{RR}$ & $\mathrm{R} / \mathrm{r}$ & $\mathrm{R} / \mathrm{r}$ & RR & $\mathrm{R} / \mathrm{r}$ & $\mathrm{RR}$ & $\mathrm{RR}$ & $\mathrm{RR}$ & $\mathrm{RR}$ & RR \\
\hline ON-25-99 & $\mathrm{RR}$ & $\mathrm{RR}$ & $\mathrm{RR}$ & $\mathrm{rr}$ & $\mathrm{rr}$ & $\mathrm{RR}$ & RR & $\mathrm{RR}$ & $\mathrm{RR}$ & $\mathrm{RR}$ & $\mathrm{R} / \mathrm{r}$ & $\mathrm{RR}$ & $\mathrm{RR}$ & $\mathrm{RR}$ & $\mathrm{RR}$ & $\mathrm{RR}$ & $\mathrm{RR}$ & $\mathrm{RR}$ \\
\hline ON-43-99 & $\mathrm{R} / \mathrm{r}$ & $\mathrm{RR}$ & $\mathrm{R} / \mathrm{r}$ & $\mathrm{R} / \mathrm{r}$ & $\mathrm{rr}$ & $\mathrm{R} / \mathrm{r}$ & $\mathrm{R} / \mathrm{r}$ & $\mathrm{R} / \mathrm{r}$ & $\mathrm{R} / \mathrm{r}$ & $\mathrm{R} / \mathrm{r}$ & $\mathrm{R} / \mathrm{r}$ & $\mathrm{R} / \mathrm{r}$ & $\mathrm{R} / \mathrm{r}$ & $\mathrm{R} / \mathrm{r}$ & $\mathrm{R} / \mathrm{r}$ & $\mathrm{R} / \mathrm{r}$ & $\mathrm{R} / \mathrm{r}$ & $\mathrm{R} / \mathrm{r}$ \\
\hline ON-45-99 & $\mathrm{R} / \mathrm{r}$ & $\mathrm{R} / \mathrm{r}$ & $\mathrm{R} / \mathrm{r}$ & $\mathrm{R} / \mathrm{r}$ & $\mathrm{rr}$ & $\mathrm{R} / \mathrm{r}$ & $\mathrm{R} / \mathrm{r}$ & $\mathrm{R} / \mathrm{r}$ & $\mathrm{R} / \mathrm{r}$ & $\mathrm{R} / \mathrm{r}$ & $\mathrm{R} / \mathrm{r}$ & $\mathrm{R} / \mathrm{r}$ & $\mathrm{R} / \mathrm{r}$ & $\mathrm{R} / \mathrm{r}$ & $\mathrm{R} / \mathrm{r}$ & $\mathrm{R} / \mathrm{r}$ & $\mathrm{R} / \mathrm{r}$ & $\mathrm{R} / \mathrm{r}$ \\
\hline ON-48-99 & RR & $\mathrm{RR}$ & $\mathrm{RR}$ & $\mathrm{rr}$ & $\mathrm{rr}$ & $\mathrm{RR}$ & RR & RR & RR & $\mathrm{R} / \mathrm{r}$ & $\mathrm{R} / \mathrm{r}$ & RR & $\mathrm{RR}$ & $\mathrm{RR}$ & $\mathrm{RR}$ & $\mathrm{RR}$ & $\mathrm{R} / \mathrm{r}$ & RR \\
\hline TO-41-5-1-1 & $\mathrm{RR}$ & $\mathrm{RR}$ & $\mathrm{RR}$ & $\mathrm{RR}$ & $\mathrm{RR}$ & $\mathrm{RR}$ & $\mathrm{RR}$ & $\mathrm{RR}$ & $\mathrm{RR}$ & $\mathrm{RR}$ & $\mathrm{RR}$ & $\mathrm{RR}$ & $\mathrm{RR}$ & $\mathrm{RR}$ & $\mathrm{RR}$ & $\mathrm{RR}$ & $\mathrm{rr}$ & $\mathrm{RR}$ \\
\hline TO-41-5-2-5 & RR & $\mathrm{RR}$ & $\mathrm{RR}$ & RR & $\mathrm{RR}$ & $\mathrm{R} / \mathrm{r}$ & $\mathrm{RR}$ & $\mathrm{RR}$ & $\mathrm{RR}$ & $\mathrm{RR}$ & RR & RR & $\mathrm{RR}$ & $\mathrm{RR}$ & $\mathrm{RR}$ & $\mathrm{RR}$ & $\mathrm{R} / \mathrm{r}$ & $\mathrm{RR}$ \\
\hline TO-41-5-6-4 & $\mathrm{RR}$ & $\mathrm{RR}$ & RR & RR & $\mathrm{RR}$ & $\mathrm{RR}$ & RR & RR & RR & $\mathrm{RR}$ & $\mathrm{RR}$ & RR & $\mathrm{R} / \mathrm{r}$ & $\mathrm{RR}$ & $\mathrm{RR}$ & $\mathrm{RR}$ & $\mathrm{R} / \mathrm{r}$ & RR \\
\hline TO-41-5-6-24 & $\mathrm{RR}$ & $\mathrm{RR}$ & $\mathrm{RR}$ & RR & $\mathrm{RR}$ & $\mathrm{RR}$ & RR & $\mathrm{RR}$ & $\mathrm{RR}$ & $\mathrm{RR}$ & $\mathrm{RR}$ & $\mathrm{RR}$ & $\mathrm{RR}$ & $\mathrm{RR}$ & RR & $\mathrm{RR}$ & $\mathrm{R} / \mathrm{r}$ & $\mathrm{RR}$ \\
\hline AB-74-1-3 & $\mathrm{R} / \mathrm{r}$ & $\mathrm{R} / \mathrm{r}$ & $\mathrm{R} / \mathrm{r}$ & $\mathrm{RR}$ & $\mathrm{R} / \mathrm{r}$ & $\mathrm{RR}$ & RR & $\mathrm{RR}$ & $\mathrm{R} / \mathrm{r}$ & $\mathrm{R} / \mathrm{r}$ & $\mathrm{R} / \mathrm{r}$ & $\mathrm{RR}$ & $\mathrm{RR}$ & $\mathrm{RR}$ & $\mathrm{R} / \mathrm{r}$ & $\mathrm{RR}$ & $\mathrm{R} / \mathrm{r}$ & $\mathrm{R} / \mathrm{r}$ \\
\hline AB-74-1-9 & RR & $\mathrm{RR}$ & $\mathrm{RR}$ & $\mathrm{R} / \mathrm{r}$ & $\mathrm{RR}$ & $\mathrm{RR}$ & $\mathrm{R} / \mathrm{r}$ & RR & RR & $\mathrm{R} / \mathrm{r}$ & $\mathrm{R} / \mathrm{r}$ & RR & $\mathrm{R} / \mathrm{r}$ & $\mathrm{R} / \mathrm{r}$ & $\mathrm{RR}$ & $\mathrm{RR}$ & $\mathrm{R} / \mathrm{r}$ & RR \\
\hline AB-74-1-10 & $\mathrm{R} / \mathrm{r}$ & $\mathrm{R} / \mathrm{r}$ & $\mathrm{R} / \mathrm{r}$ & $\mathrm{R} / \mathrm{r}$ & $\mathrm{R} / \mathrm{r}$ & $\mathrm{R} / \mathrm{r}$ & $\mathrm{R} / \mathrm{r}$ & $\mathrm{R} / \mathrm{r}$ & $\mathrm{R} / \mathrm{r}$ & $\mathrm{R} / \mathrm{r}$ & $\mathrm{RR}$ & $\mathrm{R} / \mathrm{r}$ & $\mathrm{R} / \mathrm{r}$ & $\mathrm{R} / \mathrm{r}$ & $\mathrm{R} / \mathrm{r}$ & $\mathrm{R} / \mathrm{r}$ & $\mathrm{R} / \mathrm{r}$ & $\mathrm{R} / \mathrm{r}$ \\
\hline AB-74-1-13 & $\mathrm{RR}$ & $\mathrm{RR}$ & $\mathrm{RR}$ & $\mathrm{R} / \mathrm{r}$ & $\mathrm{RR}$ & $\mathrm{RR}$ & RR & $\mathrm{RR}$ & $\mathrm{RR}$ & $\mathrm{RR}$ & $\mathrm{RR}$ & $\mathrm{RR}$ & $\mathrm{RR}$ & $\mathrm{RR}$ & $\mathrm{RR}$ & $\mathrm{RR}$ & $\mathrm{R} / \mathrm{r}$ & $\mathrm{RR}$ \\
\hline AB-74-1-16 & $\mathrm{R} / \mathrm{r}$ & $\mathrm{R} / \mathrm{r}$ & $\mathrm{R} / \mathrm{r}$ & $\mathrm{R} / \mathrm{r}$ & $\mathrm{R} / \mathrm{r}$ & $\mathrm{R} / \mathrm{r}$ & $\mathrm{R} / \mathrm{r}$ & $\mathrm{R} / \mathrm{r}$ & $\mathrm{RR}$ & $\mathrm{R} / \mathrm{r}$ & $\mathrm{R} / \mathrm{r}$ & $\mathrm{R} / \mathrm{r}$ & $\mathrm{R} / \mathrm{r}$ & $\mathrm{R} / \mathrm{r}$ & $\mathrm{R} / \mathrm{r}$ & $\mathrm{R} / \mathrm{r}$ & $\mathrm{R} / \mathrm{r}$ & $\mathrm{R} / \mathrm{r}$ \\
\hline AB-74-1-18 & $\mathrm{RR}$ & $\mathrm{RR}$ & RR & $\mathrm{R} / \mathrm{r}$ & $\mathrm{RR}$ & $\mathrm{RR}$ & RR & RR & RR & $\mathrm{RR}$ & $\mathrm{RR}$ & RR & $\mathrm{RR}$ & $\mathrm{RR}$ & $\mathrm{RR}$ & $\mathrm{R} / \mathrm{r}$ & $\mathrm{R} / \mathrm{r}$ & RR \\
\hline
\end{tabular}

$\mathrm{RR}$ - homozigoto resistente; $\mathrm{rr}$ - homozigoto suscetível; R/r - em segregação. 
patótipos, apresentando um padrão de resistência igual ao do progenitor Ouro Negro (Tabela 1). As demais isolinhas deste retrocruzamento segregaram para resistência a todos os patótipos de $U$. appendiculatus testados. As isolinhas derivadas dos demais retrocruzamentos (Rudá x TO, Rudá x AB 136 e Rudá x AND 277) foram suscetíveis a todos os patótipos de $U$. appendiculatus testados.

Quanto à avaliação da resistência à mancha-angular, observou-se que as isolinhas provenientes do cruzamento entre Rudá x Ouro Negro apresentaram-se suscetíveis. Já as isolinhas provenientes do cruzamento entre Rudá x AND 277 mostraram-se resistentes, sendo que a isolinha AND-7-2-94-6 comportou-se como homozigota resistente a todos os patótipos testados (Tabela 3).

Comparando-se os espectros de resistência dos progenitores doadores e das isolinhas deles derivadas podese verificar que as isolinhas TO-41-5-6-24, AB-74-1-18, ON25-99 e AND-7-2-9-4-6 apresentam essencialmente os mesmos genes de resistência presentes em seus progenitores (Tabelas $1,2$ e 3$)$.

As melhores isolinhas de cada subprograma estão sendo intercruzadas visando a piramidação de genes de resistência à ferrugem, à antracnose e à mancha-angular no background "carioca" Rudá. Além disso, isolinhas obtidas no subprograma envolvendo a cultivar Ouro Negro têm apresentado excelente desempenho, tanto em produtividade quanto em resistência a doenças, em ensaios preliminares de linhagens conduzidos na região sul do país (G.E.S. Carneiro Embrapa Soja, comunicação pessoal). As melhores isolinhas dos quatro subprogramas de retrocruzamento constituem um precioso germoplasma do qual genes de resistência a doenças podem ser facilmente mobilizados para uso em outros programas de melhoramento do feijoeiro.

TABELA 3-- Avaliação da resistência à mancha-angular em cultivares e isolinhas de feijoeiro-comum (Phaseolus vulgaris)

\begin{tabular}{lcccc}
\hline \hline \multirow{2}{*}{$\begin{array}{l}\text { Penótipo de } \\
\text { Phaseolus vulgaris }\end{array}$} & \multicolumn{4}{c}{ Patótipos de Phaeoisariopsis } \\
\cline { 2 - 5 } & $\mathbf{6 3 - 1 9}$ & $\mathbf{3 1 - 1 5}$ & $\mathbf{6 3 - 3 1}$ & $\mathbf{6 3 - 2 3}$ \\
\hline Rudá & $\mathrm{rr}$ & $\mathrm{rr}$ & $\mathrm{rr}$ & $\mathrm{rr}$ \\
Ouro Negro & $\mathrm{rr}$ & $\mathrm{rr}$ & $\mathrm{R} / \mathrm{r}$ & $\mathrm{R} / \mathrm{r}$ \\
AND 277 & $\mathrm{RR}$ & $\mathrm{RR}$ & $\mathrm{RR}$ & $\mathrm{RR}$ \\
ON-06-99 & $\mathrm{rr}$ & $\mathrm{rr}$ & $\mathrm{rr}$ & $\mathrm{rr}$ \\
ON-25-99 & $\mathrm{rr}$ & $\mathrm{rr}$ & $\mathrm{rr}$ & $\mathrm{rr}$ \\
ON-43-99 & $\mathrm{rr}$ & $\mathrm{rr}$ & $\mathrm{rr}$ & $\mathrm{rr}$ \\
ON-45-99 & $\mathrm{rr}$ & $\mathrm{rr}$ & $\mathrm{rr}$ & $\mathrm{rr}$ \\
ON-48-99 & $\mathrm{rr}$ & $\mathrm{rr}$ & $\mathrm{rr}$ & $\mathrm{rr}$ \\
AND-7-2-9-4-6 & $\mathrm{RR}$ & $\mathrm{RR}$ & $\mathrm{RR}$ & $\mathrm{RR}$ \\
AND-7-2-9-4-10 & $\mathrm{RR}$ & $\mathrm{R} / \mathrm{r}$ & $\mathrm{RR}$ & $\mathrm{RR}$ \\
AND-7-2-9-7-10 & $\mathrm{R} / \mathrm{r}$ & $\mathrm{R} / \mathrm{r}$ & $\mathrm{R} / \mathrm{r}$ & $\mathrm{R} / \mathrm{r}$ \\
\hline
\end{tabular}

*Isolados classificados por Nietsche (1997); RR - homozigoto resistente; rr - homozigoto suscetível; R/r - em segregação.

\section{AGRADECIMENTOS}

Este trabalho foi parcialmente financiado pela EMBRAPA. V.A. Ragagnin - bolsista de Mestrado do CNPq. A.L. Alzate-Marin - pesquisadora visitante com bolsa da FAPEMIG. T.L.P.O. Souza e K.M.A. Arruda - bolsistas de I.C. do CNPq.

\section{REFERÊNCIAS BIBLIOGRÁFICAS}

ALZATE-MARIN, A.L., FALEIRO, F.G., CARVALHO, G.A., BARROS, E.G. \& MOREIRA, M.A. Breeding common bean for resistance to fungal diseases assisted by molecular markers. The International Conference on the Status of Plant \& Animal Genome Research. San Diego, California. 1997. p.108.

ALZATE-MARIN, A.L., COSTA, M.R., BARROS, E.G. \& MOREIRA, M.A. Characterization of the anthracnose resistance locus present in cultivar Ouro Negro (Honduras 35). Annual Report of the Bean Improvement Cooperative 44:115-116. 2001.

ANDRADE, E.M., COSTA, J.G.C. \& RAVA, C.A. Variabilidade patogênica de isolados de Colletotrichum lindemuthianum de algumas regiões brasileiras. Anais. Reunião Nacional de Pesquisa de FeijãoRENAFE .Salvador. Embrapa Arroz e Feijão, Documento 99. 1999. pp.242-244.

BASSETT, M.J. List of genes. Annual Report of the Bean Improvement Cooperative 39:1-19, 1996.

CARRIJO, I.V., CHAVES, G.M. \& PEREIRA, A.A. Reação de vinte e cinco variedades de Phaseolus vulgaris a trinta e nove raças fisiológicas de Uromyces phaseoli var. typica Arth., em condições de casa-de-vegetação. Fitopatologia Brasileira 5:245-255. 1980.

CARVALHO, G.A., PAULA JÚNIOR, T.J., ALZATE-MARIN, A.L., NIETSCHE, S., BARROS, E.G., \& MOREIRA, M.A. Herança da resistência da linhagem AND 277 de feijoeiro-comum à raça 63.23 de Phaeoisariopsis griseola e identificação de marcador RAPD ligado ao gene de resistência. Fitopatologia Brasileira 23:482-485. 1998.

CORRÊA, R.X. Genes de resistência a doenças do feijoeiro: identificação de marcadores moleculares, organização e identificação de análogos. (Tese de Doutorado). Viçosa. Universidade Federal de Viçosa. 1999.

DAVISON, A.D. \& VAUGHAN, E.K. Effect of uredospore concentration on determination of races of Uromyces phaseoli var. phaseoli. Phytopathology 54:336-338. 1964.

FALEIRO, F.G. Identificação de raças, diversidade genética de Uromyces appendiculatus var. appendiculatus e herança da resistência no feijoeiro. (Tese de Mestrado). Viçosa. Universidade Federal de Viçosa. 1997.

FALEIRO, F.G., VINHADELLI, W.S., RAGAGNIN, V.A., ZAMBOLIM, L., PAULA JÚNIOR, T.J., MOREIRA, M.A. \& BARROS, E.G. Identificação de raças fisiológicas de Uromyces appendiculatus no estado de Minas Gerais, Brasil. Fitopatologia Brasileira 24:166-169. 1999a.

FALEIRO, F.G., RAGAGNIN, V.A., VINHADELLI, W.S., PAULA JÚNIOR, T.J., MOREIRA, M.A. \& BARROS, E.G. Resistência de variedades de feijoeiro-comum a quatro raças de Uromyces appendiculatus. Revista Ceres 46:11-18. 1999b.

FALEIRO, F.G., RAGAGNIN, V.A., CORRÊA, R.X., VINHADELLI, W.S., MOREIRA, M.A., \& BARROS, E.G. Ligação gênica da resistência à ferrugem e à antracnose na variedades de feijão 
Ouro Negro. Revista Ceres 47:375-382. 2000.

FALEIRO, F.G., RAGAGNIN, V.A., CARVALHO, G.A., PAULA JÚNIOR, T.J., MOREIRA, M.A. \& BARROS, E.G. Development of common bean lines resistant to rust and anthracnose by molecular marker-assisted backcrossing. Annual Report of the Bean Improvement Cooperative. 44:109-110. 2001a.

FALEIRO, F.G., NIETSCHE, S., RAGAGNIN, V.A., BORÉM, A., MOREIRA, M.A. \& BARROS, E.G. Resistência de cultivares de feijoeiro-comum à ferrugem e à mancha-angular em condições de casa de vegetação. Fitopatologia Brasileira 26:86-89. 2001b.

FARIA, J.C., DEL PELOSO, M.J., CARNEIRO, G.E.S. Resistência de cultivares de feijoeiro ao vírus do mosaico comum necrótico. Fitopatologia Brasileira 25(2):197-199. 2000.

HITTALMANI, S., PARCO, A., MEW, T.V., ZEIGLER, R.S. \& HUANG, N. Fine mapping and DNA marker-assisted pyramiding of the three major genes for blast resistance in rice. Theoretical and Applied Genetics 7:1121-1128. 2000.

LANZA, M.A., PAULA JR., T.J., VINHADELLI, W.S., MORANDI, M.A.B., BARROS, E.G. \& MOREIRA, M.A. Resistência à antracnose em cultivares de feijoeiro-comum recomendadas para Minas Gerais. Fitopatologia Brasileira 22:560562. 1997.

NIETSCHE, S. Identificação de raças de Phaeoisariopsis griseola e determinação de fontes de resistência em Phaseolus vulgaris. (Tese de Mestrado). Viçosa. Universidade Federal de Viçosa. 47p. 1997.
PIO-RIBEIRO, G. \& CHAVES, G.M. Raças fisiológicas de Colletotrichum lindemuthianum (Sacc. et Magn.) Scrib. que ocorrem em alguns municípios de Minas Gerais, Espírito Santo e Rio de Janeiro. Experientiae 19:95-118. 1975.

RAVA, C.A., MOLINA, J., KAUFFMANN, M., \& BRIONES, I. Determinación de razas fisiológicas de Colletotrichum lindemuthianum en Nicaragua. Fitopatologia Brasileira 18:388-391. 1993.

RAVA, C.A., PURCHIO, A., \& SARTORATO, A. Caracterização de patótipos de Colletotrichum lindemuthianum que ocorrem em algumas regiões produtoras de feijoeiro comum. Fitopatologia Brasileira 19:167-172. 1994.

SCHWARTZ, H.F., PASTOR-CORRALES, M.A. \& SINGH, S.P. New source of resistance to anthracnose and angular leaf spot of beans (Phaseolus vulgaris L. ) Euphytica 31:741-754. 1982.

STAVELY, J.R., FREYTAG, G.F., STEADMAN, J.R. \& SCHWARTZ, H.F. The 1983 Bean Rust Workshop. Annual Report of the Bean Improvement Cooperative 26:iv-vi. 1983.

THOMAZELLA, C., GONÇALVES-VIDIGAL, M.C., VIDA, J. B., VIDIGAL FILHO, P.S. \& RIMOLDI, F. Identification of Colletotrichum lindemuthianum races in Phaseolus vulgaris L. Annual Report of the Bean Improvement Cooperative 43:82-83. 2000.

VAN SCHOONHOVEN, A. \& PASTOR-CORRALES, M.A. (comps.). Standard system for evaluation of bean germplasm. (CIAT) Cali, Colômbia. 1987. 